Fetal Diagnosis and Therapy
Fetal Diagn Ther 2019;46:274-284

DOI: $10.1159 / 000499580$
Received: October 18, 2018

Accepted after revision: March 11, 2019

Published online: May 8, 2019

\title{
Can Fetal Growth Velocity and First Trimester Maternal Biomarkers Improve the Prediction of Small-for-Gestational Age and Adverse Neonatal Outcome?
}

\author{
Manouk L.E. Hendrix ${ }^{a}$ Judith A.P. Bons ${ }^{b}$ Roy R.G. Snellings ${ }^{a}$ Otto Bekers ${ }^{b}$ \\ Sander M.J. van Kuijk ${ }^{c}$ Marc E.A. Spaanderman ${ }^{a}$ Salwan Al-Nasiry ${ }^{a}$ \\ a Department of Obstetrics and Gynecology, GROW School of Oncology and Developmental Biology, Maastricht \\ University Medical Centre (MUMC), Maastricht, The Netherlands; ${ }^{b}$ Central Diagnostic Laboratory, Maastricht \\ University Medical Centre (MUMC), Maastricht, The Netherlands; ${ }^{\mathrm{C}}$ Department of Clinical Epidemiology and Medical \\ Technology Assessment (KEMTA), Maastricht University Medical Centre (MUMC), Maastricht, The Netherlands
}

\section{Keywords}

Biochemical markers · Fetal biometry •

Fetal growth restriction $\cdot$ Placenta

\begin{abstract}
Background and Objectives: The aim of this study was to evaluate the value of adding fetal growth velocity and first trimester maternal biomarkers to baseline screening, for the prediction of small-for-gestational age (SGA) and adverse neonatal outcomes. Method: A retrospective cohort study was conducted of singleton pregnancies in the Maastricht University Medical Centre between 2012 and 2016. The biomarkers PAPP-A, $\beta$-hCG, PIGF, and sFlt-1 were measured at 11-13 weeks of gestational age (GA) and two fetal growth scans were performed (18-22 and 30-34 weeks of GA). Differences in biomarkers and growth velocities were compared between appropriate-for-gestational age (AGA; birth weight percentile 10-90) and SGA (birth weight percentile
\end{abstract}

\begin{tabular}{ll}
\hline KARGER & ( 2019 The Author(s) Karger \\
& Published by S. Karger AG, Basel Open access \\
E-Mail karger@karger.com & This article is licensed under the Creative Commons Attribution- \\
www.karger.com/fdt & $\begin{array}{l}\text { NonCommercial-NoDerivatives 4.0 International License (CC BY- } \\
\text { NC-ND) (http://www.karger.com/Services/OpenAccessLicense). } \\
\text { Usage and distribution for commercial purposes as well as any dis- } \\
\text { tribution of modified material requires written permission. }\end{array}$
\end{tabular}

$<10)$. Combinations of the biomarkers and fetal growth velocity were added to baseline screening for the prediction of SGA and adverse neonatal outcome. Results: We included 296 singleton pregnancies. Compared to AGA $(n=251)$, SGA neonates $(n=45)$ had significantly lower growth velocities in the abdominal circumference (mm/week): $10.1 \pm 0.98$ versus $10.8 \pm 0.98, p=0.001$. Compared with AGA, the SGA neonates had higher sFlt-1 multiples of the median (MoM): 0.89 (0.55) versus $0.76(0.44), p=0.023$, and a higher sFlt-1/PIGF MoM ratio: 1.09 (1.03) versus $0.90(0.64), p=0.027$. For a $15 \%$ false-positive rate, the prediction of SGA neonates increased from $44.8 \%$ for the baseline screening model to $56.5 \%$ after the addition of fetal growth velocities, and to $73.9 \%$ after the further addition of maternal biomarkers (PPV 9.6\%, NPV 82.4\%). The corresponding AUC for the three models were $0.722,0.804$, and 0.839 , respectively. In addition, AGA neonates with reduced fetal growth velocity had more adverse neonatal outcomes compared to the AGA reference group (12.4 vs. $3.9 \%, p=0.013$ ). Conclusions: Combining fetal
Manouk Hendrix, MD

Department of Obstetrics and Gynecology, GROW School of Oncology and Developmental Biology, Maastricht University Medical Centre (MUMC) PO Box 5800, NL-6202 AZ Maastricht (The Netherlands)

E-Mail manouk.hendrix@ mumc.nl 
growth velocity with first trimester biomarkers resulted in a better prediction of SGA compared to baseline screening parameters alone. This approach could possibly result in reduced adverse neonatal outcomes in neonates, who are at a potential risk due to late mild placental dysfunction.

(C) 2019 The Author(s)

Published by S. Karger AG, Basel

\section{Introduction}

Undetected fetal growth restriction (FGR) is a major cause of perinatal morbidity. Fetuses with FGR have an increased risk of various complications, such as perinatal death, impaired neurodevelopment, and several handicaps. In adult life they have an increased risk of manifestation of the metabolic syndrome, type 2 diabetes, coronary heart disease, and hypertension [1-8]. Antenatal diagnosed FGR is associated with substantially reduced risks of adverse outcome compared to FGR diagnosed only after birth [9]. Despite advances in fetal ultrasound scanning techniques, early detection of FGR is still difficult. An expected birth weight below the 10th percentile can be used to define small-for-gestational age (SGA) fetuses. However, the SGA group contains fetuses with FGR but also constitutionally small fetuses. What makes it even harder is that some fetuses in the appropriate-forgestational age (AGA) group decline in growth velocity and have a form of FGR that falls outside of the contemporary definition and is thus unrecognized.

Currently, the criteria for diagnosing FGR are mainly based on the estimated fetal weight and Doppler hemodynamic $[10,11]$. Routine third trimester biometry measurements could improve the detection rate of FGR in the SGA and AGA group [12]. There is histological evidence that in SGA fetuses impaired placentation due to inadequate trophoblastic invasion of the maternal spiral arteries has taken place [13-15]. Inadequate trophoblastic invasion will lead to insufficient remodelling of the spiral arteries and this leads to a placental bed still susceptible for sympathetic stimuli and, with it, decreased perfusion. Angiogenic factors such as placental growth factor (PlGF) are believed to regulate the above-mentioned process [16]. PlGF and its inhibitor, the anti-angiogenic soluble fms-like tyrosine kinase-1 (sFlt-1), play an important role in the angiogenesis and placental development, with low concentrations of PlGF and high concentrations of sFlt-1 seen in SGA fetuses [17-21]. In addition, beta-human chorionic gonadotropin ( $\beta$-hCG) and pregnancy-associated plasma protein A (PAPP-A) are also associated with

Fetal Growth Parameters and Biomarkers for the Detection of Reduced Growth pre-eclampsia, but have also been investigated regarding their association with SGA $[22,23]$.

PlGF belongs to the vascular endothelial growth factor family. It is expressed by trophoblast cells, placental villi, and human umbilical vein endothelial cells. In early placental vascular development PlGF plays a role in potent pro-angiogenic effects. Reduced placental perfusion and subsequent placental bed hypoxia may be associated with a low maternal plasma concentration of PlGF and delivery of a neonate with FGR [24]. sFlt-1 is a splice variant of the vascular endothelial growth factor receptor Flt1 and a potent inhibitor of vascular endothelial growth factor. PAPP-A is a metalloprotease in the insulin-like growth factor (IGF) system. PAPP-A increases IGF bioavailability through regulated cleavage of IGF-binding protein 4 (IGFBP4) and is therefore a critical growth regulatory factor during fetal development [25]. Low PAPPA levels are associated with chromosomal anomalies in fetuses, but can also be used to predict adverse pregnancy outcomes such as FGR [26].

Many studies describe the role of PlGF, sFlt-1, and PAPP-A in the detection of pre-eclampsia, but their role in the prediction of FGR is less clear. Recent studies demonstrate that pregnancies resulting in the birth of SGA infants show similarly decreased levels of maternal PlGF and PAPP-A at $11+0$ to $13+6$ weeks of GA [22, 27-29]. There is also an association seen between FGR and lower PAPP-A and $\beta$-hCG values at 8-14 weeks of GA [26]. In addition, a high sFlt-1/PlGF ratio in pregnant women is associated with adverse neonatal outcomes [30]. However, the difference between SGA and FGR in the biomarker profile is still unclear. In the present study, we present differences between the categories of birth weight percentiles in the maternal biochemical biomarkers: PAPP-A, $\beta$-hCG, PlGF, and sFlt- 1 , all assessed at the first trimester of pregnancy and ultrasound growth velocity parameters of the second and third trimester. We also combined the maternal biochemical biomarkers with ultrasound growth parameters. The purpose of this study was to identify to what extent these parameters are able to detect intrauterine growth restriction and adverse neonatal outcome in SGA and AGA neonates.

\section{Materials and Methods}

Study Design and Participants

In this retrospective cohort study pregnant women were included at the Maastricht University Medical Centre during the period of October 2012 to June 2016. Women were only included in this cohort if they had had a singleton pregnancy without congen- 
ital anomalies, measurements of PAPP-A, $\beta$-hCG, PlGF, and sFlt1 at 11-13 weeks, and if at least two fetal growth scans were available (one between 18 and 22 weeks of GA and one between 30 and 34 weeks of GA).

The pregnancies were dated according to the formula of Robinson calculated from the crown-rump length measurement in the first trimester [31]. We excluded women who had an infant with a birth weight above the 90th percentile (large-for GA) because we wanted to predict adverse outcome. We defined a "normal" reference group with appropriate for GA birth weights. Patients were informed about the research at this university medical centre, and were given information about the data collection from their records for research purpose. Patients who refused to allow the use of their records were excluded from the study. The study protocol was approved by the medical ethical committee of the Maastricht University Medical Centre (17-4-0.15.1/ab). All procedures were in accordance with institutional guidelines and adhered to the principles of the Declaration of Helsinki and Title 45, US Code of Federal Regulations, Part 46, Protection of Human Subjects (revised November 13, 2001, effective December 13, 2001). Consent to participate was waived because of the retrospective study design.

\section{Sampling of Blood Serum}

For all patients, blood samples were collected at week 11-13 of GA. All blood biomarkers (PlGF, sFlt-1, PAPP-A, and $\beta$-hCG) were measured in serum. Samples were analysed in a certified laboratory using commercially available kits. Measurements of sFlt-1 and PIGF assays were performed according to the manufacturer's instructions on the fully automated BRAHMS KRYPTOR compact PLUS system (ThermoFisher Scientific, Hennigsdorf, Germany). Both assays are immunometric assays based on Time Resolved Amplified Cryptate Emission (TRACE) technology as described elsewhere [32]. Measurements of PAPP-A and free $\beta$-hCG were performed according to the manufacturer's instructions on the (fully automated) Auto-Delfia system (PerkinElmer, Turku, Finland). Both assays are fluoroimmunometric assays.

\section{Data Analysis}

Maternal data were collected during routine hospital visits. Maternal age, height, weight, smoking status, and medical and obstetric history were recorded. Multiples of the median (MoM) values were calculated according to the GA at measurement.

Ultrasound measurements of abdominal circumference (AC), biparietal diameter (BPD), head circumference (HC), and femur length (FL) were recorded by an experienced sonographist, using a GE Voluson ultrasound machine, with a 2- to $5-\mathrm{MHz}$ curved-array transducer, in accordance with the routine mid-trimester fetal ultrasound scan guidelines [33]. These fetal growth scans were performed in two periods, one around 20 weeks (18-22 weeks) of GA during the second trimester anomaly scan, and one around 32 weeks (30-34 weeks) as a routine third trimester growth scan $[34,35]$. Fetal growth parameters were recorded in an electronic database (Astraia $\mathrm{GmbH})$. The estimated fetal weight was calculated using the Hadlock equation [36] (Hadlock C; $\log _{10} \mathrm{BW}=1.335-0.0034[\mathrm{AC}][\mathrm{FL}]+$ $0.0316[\mathrm{BPD}]+0.0457[\mathrm{AC}]+0.1623[\mathrm{FL}])$. For the purpose of this article we calculated velocities of the fetal growth parameters (in $\mathrm{mm}$ /week) as the difference in absolute value of the measured parameters between the two examination periods, divided by the number of weeks.
Birth weight was quantified as percentiles according to the Dutch reference standard [37]. The percentiles were divided into two categories: (1) birth weight percentiles $<10$ as the SGA group, and (2) birth weight percentiles $10-90$ as the AGA group.

The SGA and AGA fetuses were stratified into subgroups. The group with a reduced growth velocity of more than $20 \%$ in the AC velocity between 20 and 30 weeks were classified as fetuses with reduced velocity.

Potential predictors of SGA were tested in three models. The first model includes the baseline screening: maternal age, conception method (spontaneous vs. assisted), smoking during pregnancy (yes/no), previous SGA (yes/no), previous SGA stillbirth (yes/ no), previous pre-eclampsia (yes/no), maternal BMI, first-trimester free $\beta$-hCG, first-trimester PAPP-A, AC at 32 (30-34) weeks of GA. The second model consists of the baseline screening (first model) and fetal growth velocities (mm/week) of AC (ACv), BPD (BPDv), $\mathrm{HC}(\mathrm{HCv})$, and FL (FLv) between 20 and 32 weeks of GA. The third model consists of model 2, with the addition of MoM serum PlGF, sFlt-1, and sFlt-1/PlGF ratio measured at 12 weeks of GA.

\section{Neonatal Dato}

Delivery and neonatal outcomes were registered by the obstetrician or in case of an adverse outcome by the paediatrician. APGAR scores were assessed at 1 and 5 min after birth. We used a cut-off of less than 7 at 5 min to determine adverse outcome [38]. Metabolic acidosis was defined as an umbilical artery blood $\mathrm{pH}$ $<7.0$ and base deficit $>12 \mathrm{mmol} / \mathrm{L}$ [39]. Neonatal hypoglycaemia was defined as a plasma glucose level of less than $30 \mathrm{mg} / \mathrm{dL}(1.65$ $\mathrm{mmol} / \mathrm{L})$ in the first $24 \mathrm{~h}$ of life and less than $45 \mathrm{mg} / \mathrm{dL}(2.5$ $\mathrm{mmol} / \mathrm{L})$ thereafter. The composite adverse neonatal outcome consists of 4 complications: asphyxia, sepsis, respiratory distress syndrome [40], and transient tachypnoea of the new born [41]. These complications were recorded in medical files and were defined according to the judgments of the attending medical staff. Asphyxia was defined as intrapartum-related hypoxia-ischemia with multiple organ failure including encephalopathy [42]. Additionally, we recorded the neonatal stay at the intensive care unit.

\section{Statistical Analysis}

Baseline characteristics of the study sample were quantified as means with standard deviations (SD), median with range, and percentages with counts. Comparisons between the groups were made using the independent $t$ test or Mann-Whitney $\mathrm{U}$ test for continuous data. For comparing categorical data between groups, the $\chi^{2}$ test was performed. Differences in circulating levels of biomarkers between the three groups were tested using one-way analysis of variance (ANOVA). Post hoc tests were used to compare each two groups. Next, we computed odds ratios (OR), with upper and lower limits of $95 \%$ confidence intervals (CI), and the respective $p$ values were estimated separately for each potential predictor for SGA using logistic regression analysis. Second, each potential predictor was combined together in a multivariable logistic regression model. All the ORs were adjusted for GA, maternal age, BMI, parity, and smoking status. The area under the receiver operating characteristic curve (AUC) was used to assess the discriminative ability of each factor. We tested the three models: baseline screening (model 1), with the addition of fetal growth velocities (model 2), and with the addition of both fetal growth velocities and biomarkers (model 3). The predicted probabilities from each regression 
Table 1. Baseline characteristics of the total cohort, reference group (birth weight percentiles 10-90), and study group (birth weight percentiles $<10$ )

\begin{tabular}{|c|c|c|c|c|}
\hline & $\begin{array}{l}\text { Total study cohort } \\
(n=296)\end{array}$ & $\begin{array}{l}\text { AGA: } \\
\text { reference group } \\
(n=251)\end{array}$ & $\begin{array}{l}\text { SGA: } \\
\text { study group } \\
(n=45)\end{array}$ & $p$ value \\
\hline Maternal age, years & $32.7 \pm 4.6$ & $32.8 \pm 4.5$ & $32.0 \pm 4.7$ & 0.225 \\
\hline Primiparous, \% & $49.3(146 / 296)$ & $46.6(117 / 251)$ & $64.4(29 / 45)$ & 0.017 \\
\hline BMI & $23.9 \pm 4.6$ & $24.1 \pm 4.8$ & $23.1 \pm 3.7$ & 0.212 \\
\hline Smoking, \% (yes/total) & $15.0(40 / 267)$ & $14.2(32 / 225)$ & $19.0(8 / 42)$ & 0.108 \\
\hline GA at birth, days & $273[79]$ & $274[79]$ & $273[79]$ & 0.087 \\
\hline GA $<34$ weeks, $\%$ & $1.7(5 / 296)$ & $1.2(3 / 251)$ & $4.4(2 / 45)$ & 0.167 \\
\hline GA 34-36 weeks, \% & $5.1(15 / 296)$ & $4.4(11 / 251)$ & $8.9(4 / 45)$ & 0.258 \\
\hline GA > 36 weeks, $\%$ & $93.2(276 / 296)$ & $9.4(237 / 251)$ & $86.7(39 / 45)$ & 0.097 \\
\hline $\mathrm{AC}<10$ th percentile at 20 weeks GA & $4.0(12 / 296)$ & $3.2(8 / 251)$ & $8.8(4 / 45)$ & 0.074 \\
\hline $\mathrm{AC}<10$ th percentile at 30 weeks GA & $9.1(27 / 296)$ & $4.8(12 / 251)$ & $33.3(15 / 45)$ & $<0.001$ \\
\hline Birth weight, g & $3,061.1 \pm 547.8$ & $3,186.4 \pm 460.76$ & $2,389.24 \pm 518.7$ & $<0.001$ \\
\hline Percentile & $38 \pm 26.8$ & $44 \pm 24.9$ & $6.0 \pm 2.9$ & $<0.001$ \\
\hline Previous SGA & $13.2(39 / 296)$ & $13.9(35 / 251)$ & $8.9(4 / 45)$ & 0.356 \\
\hline Previous SGA stillbirth & $0.7(2 / 296)$ & $0.8(2 / 251)$ & $0(0 / 45)$ & 0.548 \\
\hline Previous PE & $1.7(5 / 296)$ & $2.0(5 / 251)$ & $0(0 / 45)$ & 0.340 \\
\hline $\mathrm{PE}, \%$ & $2.7(8 / 296)$ & $1.6(4 / 251)$ & $8.9(4 / 45)$ & 0.021 \\
\hline Induction of labour, \% & $32.4(96 / 296)$ & $31.1(78 / 251)$ & $40.0(18 / 45)$ & 0.299 \\
\hline \multicolumn{5}{|l|}{ Mode of delivery, $\%$} \\
\hline Vaginal & $84.8(251 / 296)$ & $87.3(219 / 251)$ & $71.1(32 / 45)$ & 0.006 \\
\hline Prelabour caesarean & $8.4(25 / 296)$ & $7.6(19 / 251)$ & $13.3(6 / 45)$ & 0.122 \\
\hline Intrapartum caesarean & $6.8(20 / 296)$ & $5.2(13 / 251)$ & $15.6(7 / 45)$ & 0.027 \\
\hline NICU stay, \% (yes/total) & $5.7(16 / 282)$ & $11.3(12 / 106)$ & $11.1(4 / 36)$ & 0.093 \\
\hline 5 -min APGAR $<7, \%$ & $1.4(4 / 291)$ & $3.8(4 / 106)$ & $0(0 / 45)$ & 0.645 \\
\hline Metabolic acidosis, \% & $6.2(14 / 225)$ & $6.7(13 / 194)$ & $3.2(1 / 31)$ & 0.699 \\
\hline Hypoglycaemia, \% & $11.3(32 / 282)$ & $9.8(24 / 246)$ & $22.2(8 / 36)$ & 0.067 \\
\hline Adverse neonatal outcome, $\%$ & $7.8(22 / 282)$ & $7.2(18 / 251)$ & $11.1(4 / 36)$ & 0.756 \\
\hline
\end{tabular}

Data are given as the mean $\pm \mathrm{SD}, \%(n / N)$, or median [IQR] BMI, body mass index; GA, gestational age; AC, abdominal circumference; SGA, small-for-gestational age; PE, pre-eclampsia; NICU, neonatal intensive care unit. Hypoglycaemia: a plasma glucose level $<30 \mathrm{mg} / \mathrm{dL}(1.65 \mathrm{mmol} / \mathrm{L})$ in the first $24 \mathrm{~h}$ of life and $<45 \mathrm{mg} / \mathrm{dL}(2.5$ $\mathrm{mmol} / \mathrm{L}$ ) thereafter. Metabolic acidosis: umbilical artery blood $\mathrm{pH}<7.0$ and base deficit $>12 \mathrm{mmol} / \mathrm{L}$. Composite adverse neonatal outcome consists of 4 complications: asphyxia, sepsis, respiratory distress syndrome, and transient tachypnoea of the new born. Asphyxia: intrapartum-related hypoxia-ischemia with multiple organ failure including encephalopathy.

model were saved as separate variables and their accuracy was assessed using receiver-operating characteristics (ROC) curve analysis. All analyses were performed using SPSS Statistics 23 (IBM Corp, Armonk, NY, USA). $p$ values $\leq 0.05$ were considered to indicate statistical significance.

\section{Results}

\section{Participants}

Between October 2012 and June 2016, we included 296 singleton pregnant women. The sample was divided into two categories: birth weight percentile $<10(n=45)$ as
SGA, and birth weight percentile 10-90 $(n=251)$ as AGA. The general characteristics of the sample are given in Table 1 . Women had a mean age of $32.7 \pm 4.6$ years and a mean body mass index of $23.9 \pm 4.6,49.3 \%$ were primiparous and $15.0 \%$ were smokers. Pre-eclampsia was present in $2.7 \%$ of the women in the total cohort. There were no statistically significant differences in GA at delivery, induction of labour, adverse neonatal outcome, or the proportion of neonates with an APGAR $<7$ at 5 min.

The SGA group had fewer vaginal deliveries (71.1 vs. $87.3 \%, p=0.006)$, more intrapartum emergency caesarean sections ( 15.6 vs. $5.2 \%, p=0.027$ ), and more neonatal 
Table 2. Maternal serum biomarker levels and fetal growth velocities of the total cohort, reference group (birth weight percentiles 10-90) and study group (birth weight percentiles $<10$ )

\begin{tabular}{lcccc}
\hline & $\begin{array}{l}\text { Total study cohort } \\
(n=296)\end{array}$ & $\begin{array}{l}\text { AGA: } \\
\text { reference group } \\
(n=251)\end{array}$ & $\begin{array}{l}\text { SGA: } \\
\text { study group } \\
(n=45)\end{array}$ & $p$ value \\
\hline PAPP-A, mU/L & $2,481 \pm 1,580.5$ & $2,200[1,804]$ & $2,001[2,010]$ & 0.209 \\
PAPP-A MoM & $1.0 \pm 0.60$ & $0.93[4]$ & $0.93[2]$ & 0.245 \\
$\beta$-hCG, mU/L & $46.3 \pm 31.73$ & $36.8[241.99]$ & $41.0[148.61]$ & 0.682 \\
$\beta$-hCG MoM & $1.28 \pm 0.86$ & $1.04[7.11]$ & $1.16[3.85]$ & 0.686 \\
PlGF, pg/mL & $34.73 \pm 15.97$ & $31.5[99.60]$ & $29.7[115.80]$ & 0.483 \\
PlGF MoM & $0.87 \pm 0.40$ & $0.78[0.43]$ & $0.74[0.43]$ & 0.483 \\
sFlt-1, pg/mL & $1,123 \pm 544.1$ & $969[565.5]$ & $1,140[703.1]$ & 0.031 \\
sFlt-1 MoM & $0.87 \pm 0.43$ & $0.76[0.44]$ & $0.89[0.55]$ & 0.023 \\
sFlt-1/PlGF & $38.39 \pm 26.03$ & $30.66[21.76]$ & $37.06[35.15]$ & 0.033 \\
sFlt-1/PlGF MoM & $1.11 \pm 0.77$ & $0.90[0.64]$ & $1.09[1.03]$ & 0.027 \\
ACv, mm/week & $10.78 \pm 0.98$ & $10.82 \pm 0.98$ & $10.08 \pm 0.98$ & 0.001 \\
BPDv, mm/week & $3.00 \pm 0.29$ & $3.02 \pm 0.28$ & $2.78 \pm 0.29$ & $<0.001$ \\
HCv, mm/week & $10.48 \pm 0.82$ & $10.53 \pm 0.80$ & $10.01 \pm 0.83$ & 0.001 \\
HLv, mm/week & $2.47 \pm 0.23$ & $2.48 \pm 0.22$ & $2.34 \pm 0.24$ & 0.002 \\
\hline
\end{tabular}

Data are given as the mean \pm SD or median [IQR]. PAPP-A, pregnancy-associated plasma protein A; $\beta$-hCG, beta human chorionic gonadotropin; PlGF, placental growth factor; sFlt-1, soluble fms-like tyrosine kinase 1; MoM, multiples of the median, adjusted for $\mathrm{GA}$; $\mathrm{ACv}$, abdominal circumference velocity; $\mathrm{HCv}$, head circumference velocity; BPDv, biparietal diameter velocity; FLv, femur length velocity.

Table 3. Univariate OR and multivariate adjusted OR for the prediction of SGA using various parameters

\begin{tabular}{lllll}
\hline & $\begin{array}{l}\text { Univariate } \\
\text { OR }(95 \% \mathrm{CI}): \text { SGA }\end{array}$ & $p$ value & $\begin{array}{l}\text { Multivariate adjusted } \\
\text { OR (95\% CI): SGA }\end{array}$ & $p$ value \\
\hline Baseline screening model & 0.628 & 0.767 & 0.628 & 0.767 \\
Growth velocities, mm/week & $0.034(0.001-1.015)$ & 0.051 & $0.687(0.409-1.153)$ & 0.155 \\
PlGF, pg/mL & $0.992(0.971-1.014)$ & 0.483 & $1.019(0.991-1.048)$ & 0.191 \\
sFlt-1,pg/mL & $1.001(1.000-1.001)$ & 0.036 & $1.000(0.999-1.001)$ & 0.675 \\
sFlt-1/PlGF & $1.015(1.003-1.026)$ & 0.016 & $1.021(0.999-1.044)$ & 0.062 \\
\hline
\end{tabular}

Odds ratios (OR) are adjusted for GA, maternal age, BMI, parity, and smoking status. Baseline screening model: maternal age, conception method, smoking during pregnancy, previous SGA, previous SGA stillbirth, previous PE, maternal BMI, $\beta$-hCG, PAPP-A, and abdominal circumference at 32 weeks of GA. PAPP-A, pregnancy-associated plasma protein; $\beta$-hCG, beta-human chorionic gonadotropin; PlGF, placental growth factor; sFlt-1, soluble fms-like tyrosine kinase 1 .

hypoglycaemia ( 22.2 vs. $9.8 \%, p=0.067$ ), but there was no difference in rates of admission to the NICU (7.2 vs. $11.1 \%, p=0.756)$.

\section{Differences in Serum Biomarkers and Fetal Growth Velocities}

Table 2 shows the differences in first trimester maternal serum biomarkers and fetal growth velocities between
SGA and AGA (reference group) neonates. SGA neonates, compared with AGA had higher levels of sFlt-1 (pg/mL; 1,140 [703.1] vs. 969 [565.50], $p=0.031)$, higher sFlt-1 (pg/mL) MoM (0.89 [0.55] vs. 0.76 [0.44], $p=$ 0.023 ), and a higher sFlt-1/PlGF ratio (37.06 [35.15] vs. 30.66 [21.76], $p=0.033$ ), but no significant differences in PAPP-A, PAPP-A MoM, $\beta$-hCG, $\beta$-hCG MoM, PlGF, and PlGF MoM levels. Fetal growth velocities were also 
Table 4. Predictive performance of three models for the prediction of SGA

\begin{tabular}{lccc}
\hline Predictive value & Model 1 & Model 2 & Model 3 \\
\hline AUC (95\% CI) & $0.722(0.615-0.829)$ & $0.804(0.700-0.909)$ & $0.839(0.746-0.932)$ \\
\hline $5 \%$ FPR & & & \\
Sensitivity & $31.0(16.0-51.0)$ & $47.8(27.4-68.9)$ & $43.5(23.9-65.1)$ \\
PPV & $4.6(2.3-8.8)$ & $5.7(3.0-10.1)$ & $5.3(2.7-9.7)$ \\
NPV & $33.3(17.9-52.9)$ & $45.4(25.1-67.3)$ & $40.9(21.5-63.3)$ \\
\hline $10 \%$ FPR & & & \\
Sensitivity & $41.4(24.1-60.9)$ & $56.5(34.9-76.1)$ & $7.6(38.8-79.5)$ \\
PPV & $6.3(3.5-11.1)$ & $7.0(3.9-11.9)$ & $67.9(47.6-83.4)$ \\
NPV & $54.1(37.1-70.2)$ & $65.5(45.7-81.4)$ & $73.9(51.3-88.9)$ \\
\hline $15 \%$ FPR & & $9.6(5.8-15.1)$ \\
Sensitivity & $44.8(27.0-64.0)$ & $56.5(34.9-76.1)$ & $82.4(64.8-92.6)$ \\
PPV & $7.2(4.0-12.3)$ & $7.3(4.1-12.5)$ & \\
NPV & $65.2(49.7-78.2)$ & $6.4(57.6-86.4)$ & $78.3(55.8-91.7)$ \\
\hline $20 \%$ FPR & & & $10.7(6.6-16.5)$ \\
Sensitivity & $55.2(36.0-73.0)$ & $9.4(5.6-15.1)$ & $88.4(74.1-95.6)$ \\
PPV & $9.2(5.5-14.8)$ & $84.8(70.5-93.2)$ & \\
NPV & $75.0(60.8-85.5)$ &
\end{tabular}

Data are given as \% (95\% CI) or as indicated. Model 1, baseline screening: maternal age, conception method, smoking status, BMI, previous SGA, previous SGA stillbirth, previous PE, AC (abdominal circumference $<10$ th percentile at 32 weeks of GA), $\beta$-hCG and PAPP-A at 12 weeks of GA. Model 2, baseline screening with growth velocities: ACv, HCv, BPDv, and FLv between 20 and 32 weeks of GA. Model 3: model 2 with maternal biomarkers: PlGF, sFlt-1, and sFlt-1/PlGF ratio at 12 weeks of GA. PPV, positive predictive value; NPV, negative predictive value.

significantly lower in SGA compared to AGA neonates: $\mathrm{ACv}, 10.08 \pm 0.98$ versus $10.82 \pm 0.98, p=0.001 ; \mathrm{BPDv}$, $2.78 \pm 0.29$ versus $3.02 \pm 0.28, p<0.001 ; \mathrm{HCv}, 10.01 \pm 0.83$ versus $10.53 \pm 0.80, p=0.001$, and FLv, $2.34 \pm 0.24$ versus $2.48 \pm 0.22, p=0.002$.

\section{Prediction of SGA}

The sFlt-1, sFlt-1/PlGF ratio, and growth velocities all showed significant associations with SGA (Table 3). However, these were not significant after adjustment for GA, maternal age, BMI, parity, and smoking status. The baseline screening model did not show any significant association with SGA.

The discriminative ability of the three different models as quantified by AUC is shown in Figure 1 and Table 4 . For the prediction of SGA, the baseline screening (maternal age, conception method, smoking during pregnancy, previous SGA, previous SGA stillbirth, previous pre-eclampsia, maternal BMI, $\beta$-hCG, PAPP-A, and AC at 32 weeks of GA) had an AUC of 0.722 (95\% CI 0.615-0.829). Adding growth velocities $(\mathrm{ACv}, \mathrm{BPDv}, \mathrm{HCv}, \mathrm{FLv})$ to the baseline screening improved the prediction of SGA with an AUC of 0.804 (95\% CI 0.700-0.909). Adding growth

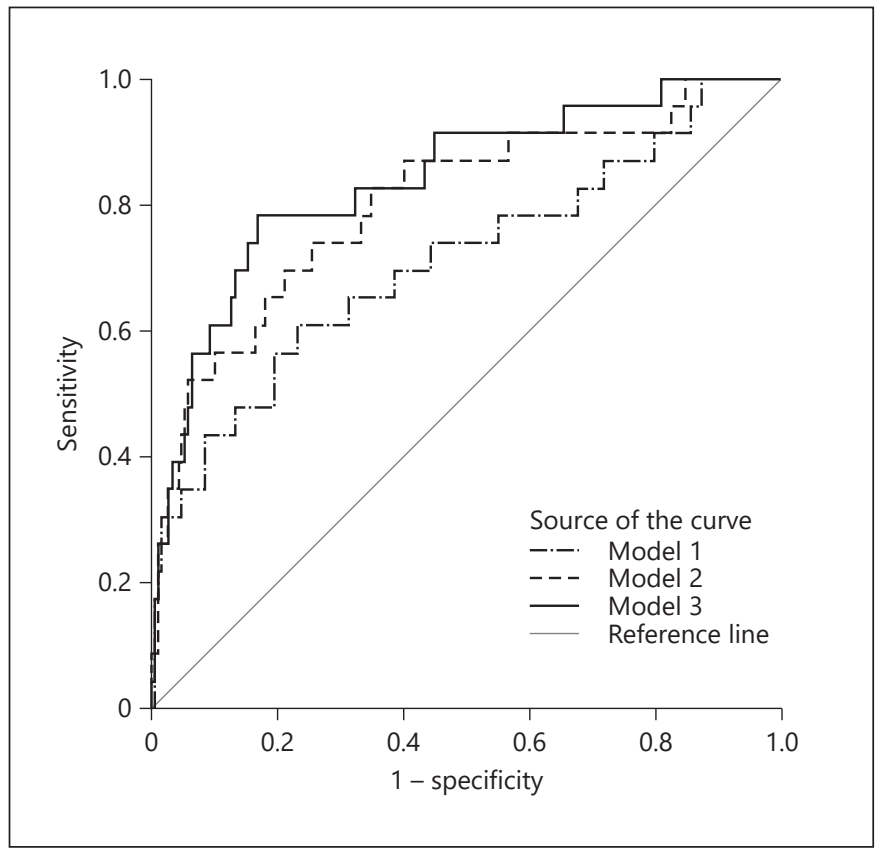

Fig. 1. ROC curve for prediction of SGA, using three different models: model 1, baseline screening; model 2, baseline screening plus fetal growth velocities; model 3, baseline screening, plus fetal growth velocities and maternal biomarkers. 
Table 5. Maternal serum biomarker levels in AGA with normal growth (reference), AGA with reduced growth velocities, SGA with normal growth, and SGA with reduced growth

\begin{tabular}{|c|c|c|c|c|c|c|c|}
\hline & $\begin{array}{l}\text { AGA normal } \\
\text { growth: } \\
\text { reference } \\
(n=154)\end{array}$ & $\begin{array}{l}\text { AGA reduced } \\
\text { growth } \\
(n=97)\end{array}$ & $p$ value & $\begin{array}{l}\text { SGA normal } \\
\text { growth } \\
(n=26)\end{array}$ & $p$ value & $\begin{array}{l}\text { SGA reduced } \\
\text { growth } \\
(n=19)\end{array}$ & $p$ value \\
\hline PAPP-A, mU/L & $2,284[2,000]$ & $1,997[1,634]$ & 0.036 & $2,199[2,431]$ & 0.405 & $1,659[2,130]$ & 0.043 \\
\hline$\beta-\mathrm{hCG}, \mathrm{mU} / \mathrm{L}$ & $39.6[34.0]$ & $36.1[27.6]$ & 0.142 & $43.9[44.0]$ & 0.476 & $23.7[29.73]$ & 0.027 \\
\hline $\mathrm{PlGF}, \mathrm{pg} / \mathrm{mL}$ & $33.2[18.95]$ & $30.8[15.2]$ & 0.067 & 30.4 [19.63] & 0.487 & $29.7[13.20]$ & 0.238 \\
\hline sFlt-1, pg/mL & $980[596.8]$ & 968 [550.9] & 0.240 & $1,140[696.2]$ & 0.533 & $1,171[913.5]$ & 0.036 \\
\hline sFlt-1/PlGF ratio & $28.8[20.8]$ & $34.6[22.9]$ & 0.818 & $37.3[39.10]$ & 0.008 & 33.9 [31.89] & 0.029 \\
\hline
\end{tabular}

Data are given as the median $[\mathrm{IQR}]$. Reduced growth is defined as a reduced growth velocity of more than $20 \%$ in the abdominal circumference velocity between 20 and 30 weeks. AGA, fetuses with a birth weight between the 10-90th percentile; SGA, fetuses with a birth weight below the 10th percentile. PAPP-A, pregnancy-associated plasma protein A; $\beta$-hCG, beta-human chorionic gonadotropin; PlGF, placental growth factor; sFlt-1, soluble fms-like tyrosine kinase 1.

Table 6. Neonatal outcome in the AGA with normal growth (reference), AGA with reduced growth velocities, SGA with normal growth, and SGA with reduced growth

\begin{tabular}{|c|c|c|c|c|c|c|c|}
\hline NICU admission (yes/total) & $3.9(6 / 154)$ & $6.2(6 / 97)$ & 0.449 & $12.5(3 / 26)$ & 0.093 & $9.5(2 / 19)$ & 0.300 \\
\hline 5 -min APGAR $<7$ & $1.3(2 / 154)$ & $2.1(2 / 97)$ & 0.617 & $0(0 / 26)$ & 0.596 & $0(0 / 19)$ & 0.644 \\
\hline Adverse neonatal outcome & $3.9(6 / 154)$ & $12.4(12 / 97)$ & 0.013 & $8.3(2 / 26)$ & 0.440 & $9.5(2 / 19)$ & 0.355 \\
\hline
\end{tabular}

Data are given as \% $(n / N)$. Hypoglycaemia: plasma glucose level $<30 \mathrm{mg} / \mathrm{dL}(1.65 \mathrm{mmol} / \mathrm{L})$ in the first $24 \mathrm{~h}$ of life and $<45 \mathrm{mg} / \mathrm{dL}(2.5$ $\mathrm{mmol} / \mathrm{L})$ thereafter. Asphyxia: intrapartum-related hypoxia-ischemia with multiple organ failure including encephalopathy. Adverse neonatal outcome: asphyxia, sepsis, respiratory distress syndrome and transient tachypnea of the newborn. NICU, neonatal intensive care unit.

velocities and biomarkers (PlGF, sFlt-1, sFlt-1/PlGF ratio) to the baseline screening improved the prediction of SGA even more, with an AUC of 0.839 (95\% CI 0.746-0.932).

For a $15 \%$ false-positive rate (FPR), the baseline screening model predicted $44.8 \%$ of SGA neonates. The addition of fetal growth velocities gave a prediction of $56.5 \%$ for SGA. The combination of baseline screening, growth velocities, and maternal biomarkers gave a prediction of 73.9\% (PPV 9.6\%, NPV 82.4\%). The predictive performance of the three models for different FPRs is shown in Table 4.

\section{Stratification of Outcome by Reduced Fetal Growth}

The two groups (AGA and SGA) were both divided into two subgroups based on $\mathrm{ACv}$ between 20 and 30 weeks: AGA with normal growth consisting of 154 (52\%) neonates, as the reference group, AGA with reduced growth comprising 97 (33\%) neonates, SGA with normal growth consisting of $26(8.8 \%)$ neonates, and SGA with reduced growth featuring $19(6.4 \%)$ neonates. SGA neonates with reduced growth showed lower PAPP-A ( $p=$ $0.043)$ and $\beta$-hCG $(p=0.027)$, and higher sFlt- $1(p=$ $0.036)$ and ratio $(p=0.029)$, compared with the AGA with normal growth (reference group). A similar trend was observed within the AGA neonates with reduced velocity. 
However, only PAPP-A was significantly different between the groups $(p=0.036)$. SGA with normal growth had a higher sFlt-1/PlGF ratio $(p=0.008)$ compared to the reference group (Table 5). AGA with reduced velocity had significantly higher adverse neonatal outcomes (12.4\%) compared with AGA with normal growth (3.9\%, $p=0.013$ ). Both categories of SGA neonates (with or without reduced AC velocity) had trends towards higher adverse neonatal outcome of 9.5 and $8.3 \%$, respectively (Table 6). The other neonatal outcomes were not significantly different. Fetal AC growth velocity had a modest predictive power for AGA with adverse neonatal outcome, with a sensitivity of $52.9 \%$ for a $20 \%$ FPR and AUC of 0.716 (0.567-0.865; Fig. 2).

\section{Discussion}

\section{Main Findings}

In this large retrospective study, we tested the additional value of first trimester maternal placental biomarkers and ultrasound fetal growth velocities to predict the birth of SGA neonates. This study showed that SGA neonates had reduced fetal growth velocities between the second and third trimester compared to AGA neonates, with an altered first trimester placental biomarker profile. The combination of the maternal biochemical biomarkers at 11-13 weeks and ultrasound growth velocities at 20-32 weeks improves the prediction of SGA neonates compared to baseline screening alone. By taking fetal growth velocities into consideration, the difference in biomarker profile was mainly seen in the subgroup of SGA with reduced velocity. Even the AGA group with reduced fetal growth had a lower PAPP-A and more adverse neonatal outcomes, compared with the AGA reference group.

\section{Strengths and Limitations}

The strength of this study lies in the fact that the population consists of all women during a certain period of time with a comprehensive registration of ultrasound, obstetric, and neonatal parameters in a single tertiary centre. All women gave birth in the MUMC and had available maternal biochemical biomarkers and ultrasound growth velocities, all at the same GA. Furthermore, the levels of the biomarkers were measured at the end of the first trimester; this is a crucial moment for therapies that can be initiated to possibly alter the course of placental dysfunction.

Third, the measurement of the ultrasound growth velocities is a new and practical method of assessing fetal

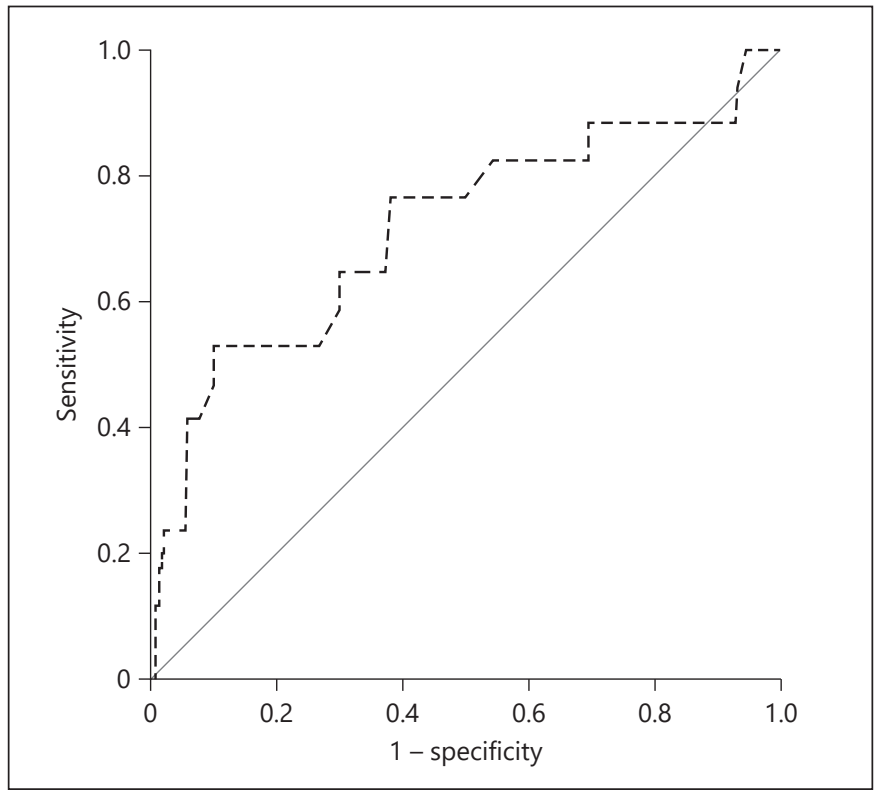

Fig. 2. ROC curve for prediction of AGA with adverse neonatal outcome, using AC growth velocity between 20 and 32 weeks of GA. The area under the ROC curve was 0.716 (0.567-0.865), with sensitivity of $52.9 \%$ and FPR of $20 \%$.

growth potential within the SGA and AGA group using a simple calculation that can be readily employed and interpreted in a low-resource setting without the need of complex mathematical models. Another strength of this study is the focus mainly on SGA without pre-eclampsia, whereas other studies gave results of SGA combined with pre-eclampsia. In this study only $2 \%$ of the population had pre-eclampsia.

We acknowledge that there are some limitations to this study. First of all, the population consists of 296 neonates, which is rather small. The results would be of greater importance in a larger population. Second, the study had a retrospective design with the inherent risk of selection bias and the exclusion of many cases in which the ultrasound examination and the biomarkers did not fall within the specified time periods. Another limitation is the heterogeneity of study population with various risk profiles for FGR and the association with pre-eclampsia, as the study was performed in a tertiary referral centre. There was inadequate recording of determinants of obstetric risk and other confounders (such as ethnicity, maternal weight and length) to enable post hoc stratification. This might limit the interpretation of our data and the generalizability to an unselected lowrisk population. 


\section{Interpretation}

The introduction of placental biomarkers (PAPP-A and $\beta$-hCG) into national aneuploidy screening programmes worldwide has soon led to the first citations linking the maternal serum levels of these biomarkers in the first trimester to a variety of obstetric complications later in pregnancy [28]. This was not surprising taking into account the fact that these biomarkers are secreted by placental cells (villous syncytiotrophoblast) into the maternal circulation and that they play a crucial role in placental development and function [43]. Since pre-eclampsia is considered an epitome of placenta dysfunction, there has been ample literature on the use of placental biomarkers to predict the occurrence of pre-eclampsia, as well as its severity and its various phenotypes.

FGR has as strong link to placental dysfunction, as does pre-eclampsia. However, the arbitrary definitions used to define both intrauterine and postnatal growth abnormalities have led to confusing conclusions regarding the link to placental abnormalities, including placental biomarkers. The data on the association between FGR and $\beta$-hCG values in the first trimester are conflicting with both lower and higher $\beta$-hCG values being reported in FGR pregnancies, possibly depending on the GA at sampling. The conclusion is that serum $\beta$-hCG is not a reliable marker to predict either FGR or pre-eclampsia; however, PAPP-A has good predictive power for both FGR and pre-eclampsia [8,26].

Many studies demonstrated that pregnancies resulting in the birth of SGA infants showed decreased PAPP-A at $11+0$ to $13+6$ weeks of GA $[22,27,29,44,45]$. Our data are consistent with these findings, showing that reduced PAPP-A and $\beta$-hCG levels were only observed in SGA pregnancies with reduced fetal growth.

The emergence of angiogenic factors as novel biomarkers of placental dysfunction has led to a surge in publications addressing their role as possibly the best biochemical predictors of pre-eclampsia, coinciding with the implementation of their use in the clinical setting of triaging women with suspected pre-eclampsia. PlGF and sFlt-1 have apposing functions on endothelial cells. Lower PIGF levels and higher sFlt- 1 levels have been linked to the development of pre-eclampsia and FGR [16, 21, 46, 47]. A ratio of sFlt-1/PlGF has been proposed to have a better predictive and discriminatory power compared to either biomarker alone, for various adverse neonatal outcomes $[30,48,49]$. Our data confirm that sFlt- 1 and the sFlt-1/PlGF ratio were higher in SGA pregnancies. Consequently, the predictive power of first trimester placental biomarkers, alone or in combination, was higher for SGA than AGA. In the subgroup of SGA neonates with normal fetal growth, the sFlt-1/PlGF ratio was higher compared to controls, suggesting general "smallness," not necessarily abnormal placentation.

It is interesting to view the differences in biomarker data between SGA and AGA with a reduced fetal growth in our population from the perspective of divergent placental pathoaetiology. Evidence suggests that there are two different phenotypes of FGR. First, an early FGR phenotype resulting in severe SGA is associated with placental bed pathology and abnormal placental development, reflected by early changes in uteroplacental Doppler profile and placental serum biomarkers. The second is a late mild FGR phenotype, characterised by mild placental disease, a normal uteroplacental Doppler profile, and abnormal hemodynamic fetal adaptation reflected by late cerebroplacental Doppler changes $[10,50]$. Our data suggest that we could identify a subgroup with reduced fetal growth velocity within AGA neonates, who had both reduced PAPP-A levels and more adverse neonatal outcomes. We therefore postulate that AGA neonates represent fetuses with normal early placentation. However, they show reduced growth velocities between the second and third trimester and subsequently fail to achieve their optimal birth weight due to external factors limiting the placental capacity of nutritional supply. This study highlights the importance of performing a third trimester biometry scan in an unselected population to determine target fetal growth. This mild and late form of restricted fetal growth is often missed in the current clinical practice and poses a challenge to obstetricians to improve its detection and reduce the associated late fetal compromise [51].

\section{Conclusion}

The detection of FGR is still a major and complicated task, as the majority with pathological restriction of growth capacity due to placental dysfunction are not properly detected. Currently, the diagnosis of FGR is mainly based on a single measurement mainly performed in the second or third trimester. To maximize the detection of FGR, we recommend combining maternal serum placental biomarkers in the first trimester such as the ones given in this study: PlGF, sFlt-1, PAPP-A, and $\beta$-hCG with fetal growth velocities. This approach could possibly result in reduced morbidity in AGA neonates, who are at a potential risk due to late mild placental dysfunction.
Hendrix/Bons/Snellings/Bekers/ van Kuijk/Spaanderman/Al-Nasiry 


\section{Statement of Ethics}

The study protocol was approved by the medical ethical committee of the Maastricht University Medical Center (17-4-0.15.1/ab). All procedures were in accordance with institutional guidelines and adhered to the principles of the Declaration of Helsinki and Title 45, US Code of Federal Regulations, Part 46, Protection of Human Subjects (revised November 13, 2001, effective December 13, 2001). Due to the retrospective study design, the consent to participate was waived. The authors have no ethical conflicts to disclose.

\section{Disclosure Statement}

The authors have no conflicts of interest to declare.

\section{Funding Sources}

There were no funding sources.

\section{Author Contributions}

M.L.E.H., S.M.J.v.K., and S.A.-N. substantially contributed to the conception and design of the study and the analysis and interpretation of data, drafted the work and revised it critically for important intellectual content, and provided final approval of the version to be published. J.A.P.B. substantially contributed to the conception and design of the study and the analysis and interpretation of data, revised the work critically for important intellectual content, and provided final approval of the version to be published. R.R.G.S. substantially contributed to the analysis and interpretation of data, drafted the work and revised it critically for important intellectual content. O.B. drafted the work and revised it critically for important intellectual content. M.E.A.S. drafted the work and revised it critically for important intellectual content, and provided final approval of the version to be published. All authors agree to be accountable for all aspects of the study in ensuring that questions relating to the accuracy or integrity of any part of the work are appropriately investigated and resolved.

\section{References}

1 M Kady S, Gardosi J; S MK. Perinatal mortality and fetal growth restriction. Best Pract Res Clin Obstet Gynaecol. 2004 Jun;18(3):397410.

2 Pallotto EK, Kilbride HW. Perinatal outcome and later implications of intrauterine growth restriction. Clin Obstet Gynecol. 2006 Jun; 49(2):257-69.

3 Lees C, Marlow N, Arabin B, Bilardo CM, Brezinka C, Derks JB, et al.; TRUFFLE Group. Perinatal morbidity and mortality in earlyonset fetal growth restriction: cohort outcomes of the trial of randomized umbilical and fetal flow in Europe (TRUFFLE). Ultrasound Obstet Gynecol. 2013 Oct;42(4):400-8.

4 Leitner Y, Fattal-Valevski A, Geva R, Eshel R, Toledano-Alhadef H, Rotstein M, et al. Neurodevelopmental outcome of children with intrauterine growth retardation: a longitudinal, 10-year prospective study. J Child Neurol. 2007 May;22(5):580-7.

5 Barker DJ. Adult consequences of fetal growth restriction. Clin Obstet Gynecol. 2006 Jun; 49(2):270-83.

6 Crispi F, Bijnens B, Figueras F, Bartrons J, Eixarch E, Le Noble F, et al. Fetal growth restriction results in remodeled and less efficient hearts in children. Circulation. 2010 Jun;121(22):2427-36.

7 Varvarigou AA. Intrauterine growth restriction as a potential risk factor for disease onset in adulthood. J Pediatr Endocrinol Metab. 2010 Mar;23(3):215-24.

8 Karagiannis G, Akolekar R, Sarquis R, Wright D, Nicolaides KH. Prediction of small-forgestation neonates from biophysical and bio- chemical markers at 11-13 weeks. Fetal Diagn Ther. 2011;29(2):148-54.

9 Lindqvist PG, Molin J. Does antenatal identification of small-for-gestational age fetuses significantly improve their outcome? Ultrasound Obstet Gynecol. 2005 Mar;25(3):258-64.

10 Figueras F, Gratacós E. Update on the diagnosis and classification of fetal growth restriction and proposal of a stage-based management protocol. Fetal Diagn Ther. 2014;36(2):86-98.

11 Figueras F, Savchev S, Triunfo S, Crovetto F, Gratacos E. An integrated model with classification criteria to predict small-for-gestational-age fetuses at risk of adverse perinatal outcome. Ultrasound Obstet Gynecol. 2015 Mar;45(3):279-85.

12 Papageorghiou AT, Ohuma EO, Altman DG, Todros T, Cheikh Ismail L, Lambert A, et al.; International Fetal and Newborn Growth Consortium for the 21st Century (INTERGROWTH-21st). International standards for fetal growth based on serial ultrasound measurements: the Fetal Growth Longitudinal Study of the INTERGROWTH-21st Project. Lancet. 2014 Sep;384(9946):869-79.

13 Sheppard BL, Bonnar J. An ultrastructural study of utero-placental spiral arteries in hypertensive and normotensive pregnancy and fetal growth retardation. Br J Obstet Gynaecol. 1981 Jul;88(7):695-705.

14 Khong TY, De Wolf F, Robertson WB, Brosens I. Inadequate maternal vascular response to placentation in pregnancies complicated by pre-eclampsia and by small-for-gestational age infants. Br J Obstet Gynaecol. 1986 Oct; 93(10):1049-59.
15 Pijnenborg R, Vercruysse L, Hanssens M. The uterine spiral arteries in human pregnancy: facts and controversies. Placenta. 2006 SepOct:27(9-10):939-58.

16 Cowans NJ, Stamatopoulou A, Matwejew E, von Kaisenberg CS, Spencer K. First-trimester placental growth factor as a marker for hypertensive disorders and SGA. Prenat Diagn. 2010 Jun;30(6):565-70.

17 Rana S, Karumanchi SA, Levine RJ, Venkatesha S, Rauh-Hain JA, Tamez H, et al. Sequential changes in antiangiogenic factors in early pregnancy and risk of developing preeclampsia. Hypertension. 2007 Jul;50(1): $137-42$.

18 Erez O, Romero R, Espinoza J, Fu W, Todem $\mathrm{D}$, Kusanovic JP, et al. The change in concentrations of angiogenic and anti-angiogenic factors in maternal plasma between the first and second trimesters in risk assessment for the subsequent development of preeclampsia and small-for-gestational age. J Matern Fetal Neonatal Med. 2008 May;21(5):279-87.

19 Taylor RN, Grimwood J, Taylor RS, McMaster MT, Fisher SJ, North RA. Longitudinal serum concentrations of placental growth factor: evidence for abnormal placental angiogenesis in pathologic pregnancies. Am J Obstet Gynecol. 2003 Jan; 188(1): 177-82.

20 Crispi F, Domínguez C, Llurba E, MartínGallán P, Cabero L, Gratacós E. Placental angiogenic growth factors and uterine artery Doppler findings for characterization of different subsets in preeclampsia and in isolated intrauterine growth restriction. Am J Obstet Gynecol. 2006 Jul;195(1):201-7.
Fetal Growth Parameters and Biomarkers for the Detection of Reduced Growth 
21 Romero R, Nien JK, Espinoza J, Todem D, Fu $\mathrm{W}$, Chung $\mathrm{H}$, et al. A longitudinal study of angiogenic (placental growth factor) and anti-angiogenic (soluble endoglin and soluble vascular endothelial growth factor receptor-1) factors in normal pregnancy and patients destined to develop preeclampsia and deliver a small for gestational age neonate. J Matern Fetal Neonatal Med. 2008 Jan;21(1): 9-23.

22 Sung KU, Roh JA, Eoh KJ, Kim EH. Maternal serum placental growth factor and pregnancy-associated plasma protein A measured in the first trimester as parameters of subsequent pre-eclampsia and small-for-gestational-age infants: A prospective observational study. Obstet Gynecol Sci. 2017 Mar;60(2):154-62.

23 Cignini P, Maggio Savasta L, Gulino FA, Vitale SG, Mangiafico L, Mesoraca A, et al. Predictive value of pregnancy-associated plasma protein-A (PAPP-A) and free beta-hCG on fetal growth restriction: results of a prospective study. Arch Gynecol Obstet. 2016 Jun; 293(6):1227-33.

24 Torry DS, Ahn H, Barnes EL, Torry RJ. Placenta growth factor: potential role in pregnancy. Am J Reprod Immunol. 1999 Jan; 41(1):79-85.

25 Conover CA, Bale LK, Overgaard MT, Johnstone EW, Laursen UH, Füchtbauer EM, et al. Metalloproteinase pregnancy-associated plasma protein A is a critical growth regulatory factor during fetal development. Development. 2004 Mar;131(5):1187-94.

26 Smith GC, Stenhouse EJ, Crossley JA, Aitken $\mathrm{DA}$, Cameron AD, Connor JM. Early pregnancy levels of pregnancy-associated plasma protein a and the risk of intrauterine growth restriction, premature birth, preeclampsia, and stillbirth. J Clin Endocrinol Metab. 2002 Apr;87(4):1762-7.

27 Thadhani R, Mutter WP, Wolf M, Levine RJ, Taylor RN, Sukhatme VP, et al. First trimester placental growth factor and soluble fms-like tyrosine kinase 1 and risk for preeclampsia. J Clin Endocrinol Metab. 2004 Feb;89(2):770-5.

28 Boucoiran I, Thissier-Levy S, Wu Y, Wei SQ, Luo ZC, Delvin E, et al.; MIROS Study Group. Risks for preeclampsia and small for gestational age: predictive values of placental growth factor, soluble fms-like tyrosine kinase-1, and inhibin A in singleton and multiple-gestation pregnancies. Am J Perinatol. 2013 Aug;30(7):607-12.

29 Tsiakkas A, Duvdevani N, Wright A, Wright D, Nicolaides KH. Serum placental growth factor in the three trimesters of pregnancy: effects of maternal characteristics and medical history. Ultrasound Obstet Gynecol. 2015 May;45(5):591-8.
30 Chang YS, Chen CN, Jeng SF, Su YN, Chen $\mathrm{CY}$, Chou HC, et al. The sFlt-1/PlGF ratio as a predictor for poor pregnancy and neonatal outcomes. Pediatr Neonatol. 2017 Dec;58(6): 529-33.

31 Robinson HP. Sonar measurement of fetal crown-rump length as means of assessing maturity in first trimester of pregnancy. BMJ. 1973 Oct; $4(5883): 28-31$.

32 Mathis G. Rare earth cryptates and homogeneous fluoroimmunoassays with human sera. Clin Chem. 1993 Sep;39(9):1953-9.

33 ISUOG [internet]. Practice guidelines for performance of the routine mid-trimester fetal ultrasound scan 2010 [cited 2018 Sept 5]. Available from: https://obgyn.onlinelibrary. wiley.com/doi/full/10.1002/uog.8831

34 Sovio U, White IR, Dacey A, Pasupathy D, Smith GC. Screening for fetal growth restriction with universal third trimester ultrasonography in nulliparous women in the Pregnancy Outcome Prediction (POP) study: a prospective cohort study. Lancet. 2015 Nov; 386(10008):2089-97.

35 Caradeux J, Eixarch E, Mazarico E, Basuki TR, Gratacos E, Figueras F. Second to third trimester longitudinal growth assessment for the prediction of small-for-gestational age and late fetal growth restriction. Ultrasound Obstet Gynecol. 2018;51(2):219-24.

36 Hadlock FP, Harrist RB, Sharman RS, Deter RL, Park SK. Estimation of fetal weight with the use of head, body, and femur measurements-a prospective study. Am J Obstet Gynecol. 1985 Feb;151(3):333-7.

37 Kloosterman GJ. [Intrauterine growth and intrauterine growth curves]. Ned Tijdschr Verloskd Gynaecol. 1969 Oct;69(5):349-65.

38 Cnattingius S, Norman M, Granath F, Petersson G, Stephansson O, Frisell T. Apgar Score Components at 5 Minutes: Risks and Prediction of Neonatal Mortality. Paediatr Perinat Epidemiol. 2017 Jul;31(4):328-37.

39 ACOG Committee on Obstetric Practice. ACOG Committee Opinion No. 348, November 2006: umbilical cord blood gas and acidbase analysis. Obstet Gynecol. 2006 Nov; 108(5):1319-22.

40 Sweet DG, Carnielli V, Greisen G, Hallman M, Ozek E, Plavka R, et al. European Consensus Guidelines on the Management of Respiratory Distress Syndrome - 2016 Update. Neonatology. 2017;111(2):107-25.

41 Buchiboyina A, Jasani B, Deshmukh M, Patole S. Strategies for managing transient tachypnoea of the newborn - a systematic review. J Matern Fetal Neonatal Med. 2017 Jul; 30(13):1524-32.
42 Morales P, Bustamante D, Espina-Marchant $\mathrm{P}$, Neira-Peña T, Gutiérrez-Hernández MA, Allende-Castro C, et al. Pathophysiology of perinatal asphyxia: can we predict and improve individual outcomes? EPMA J. 2011 Jun;2(2):211-30.

43 D’Elia P, Marzioni D, Castellucci M, Mocci C, Pala A. Homodimeric pregnancy-associated plasma protein-A in normal human placenta of first and third trimester of pregnancy: biochemical and morphological observations. Placenta. 2012 Nov;33(11):942-5.

44 Birdir C, Fryze J, Frölich S, Schmidt M, Köninger A, Kimmig R, et al. Impact of maternal serum levels of Visfatin, AFP, PAPP-A, sFlt-1 and PlGF at 11-13 weeks gestation on small for gestational age births. J Matern Fetal Neonatal Med. 2017 Mar;30(6):629-34.

45 Vandenberghe G, Mensink I, Twisk JW, Blankenstein MA, Heijboer AC, van Vugt JM. First trimester screening for intra-uterine growth restriction and early-onset pre-eclampsia. Prenat Diagn. 2011 Oct;31(10):95561.

46 Poon LC, Zaragoza E, Akolekar R, Anagnostopoulos E, Nicolaides KH. Maternal serum placental growth factor (PlGF) in small for gestational age pregnancy at $11(+0)$ to $13(+6)$ weeks of gestation. Prenat Diagn. 2008 Dec; 28(12):1110-5.

47 Litwińska E, Litwińska M, Oszukowski P, Szaflik K, Kaczmarek P. Combined screening for early and late pre-eclampsia and intrauterine growth restriction by maternal history, uterine artery Doppler, mean arterial pressure and biochemical markers. Adv Clin Exp Med. 2017 May-Jun;26(3):439-48.

48 Triunfo S, Crovetto F, Rodriguez-Sureda V, Scazzocchio E, Crispi F, Dominguez C, et al. Changes in uterine artery Doppler velocimetry and circulating angiogenic factors in the first half of pregnancies delivering a smallfor-gestational-age neonate. Ultrasound $\mathrm{Ob}$ stet Gynecol. 2017 Mar;49(3):357-63.

49 Crovetto F, Triunfo S, Crispi F, RodriguezSureda V, Roma E, Dominguez C, et al. Firsttrimester screening with specific algorithms for early- and late-onset fetal growth restriction. Ultrasound Obstet Gynecol. 2016 Sep; 48(3):340-8.

50 Savchev S, Figueras F, Sanz-Cortes M, CruzLemini M, Triunfo S, Botet F, et al. Evaluation of an optimal gestational age cut-off for the definition of early- and late-onset fetal growth restriction. Fetal Diagn Ther. 2014;36(2):99_ 105.

51 Figueras F, Gardosi J. Intrauterine growth restriction: new concepts in antenatal surveillance, diagnosis, and management. Am J Obstet Gynecol. 2011 Apr;204(4):288-300. 